\title{
Finite Element Analyses of Articular Cartilage Models Considering Depth-Dependent Elastic Modulus and Collagen Fiber Network*
}

\author{
Natsuko HOSODA**, Nobuo SAKAI***, Yoshinori SAWAE*** \\ and Teruo MURAKAMI*** \\ **Graduate School of Systems Life Sciences, Kyushu University, \\ 744 Motooka, Nishi-ku, Fukuoka-shi, Fukuoka 819-0395, Japan \\ E-mail: natsuko@tribo1.mech.kyushu-u.ac.jp \\ ***Faculty of Engineering, Kyushu University \\ 744 Motooka, Nishi-ku, Fukuoka-shi, Fukuoka 819-0395, Japan
}

\begin{abstract}
Articular cartilage has high water content and biphasic property. The structures of the tissue are inhomogeneous and anisotropic. Furthermore, the mechanical behavior of cartilage shows depth-dependence. Therefore it is necessary to consider not only the average tissue property but also the local one to explain mechanical and functional behavior. Previously, we created two-dimensional biphasic finite element (FE) cartilage tissue models considering the depth-dependence of elastic modulus distribution based on experimental results. As a result, this finding indicates that the depth-dependence of elastic modulus has a remarked influence on the deformed profile. In this study, the effectiveness of collagen fiber network in addition to the depth-dependent elastic modulus of cartilage tissue is evaluated. By creating of cartilage tissue models using axisymmetric biphasic elements and spring elements, we analyzed the unconfined compressive behaviors of articular cartilage specimens and compared the FE analyses to experimental results. Every FE model has depth-dependence of elastic modulus based on our previous formula, while the Poisson's ratio and permeability of solid phase were assumed as constant in literature data. To compare experimental result with finite element analysis (FEA), boundary conditions for FEA were given to correspond to the compression test. As a result, total load capacity and deformed profiles immediately after compression of FEA simulation on eventual model corresponded to experimental results by controlling spring constant. Furthermore, local strain of axial direction in FEA results for eventual model and experimental ones show the same tendency about time-dependent change. Then, we considered intrinsic fluid flow of eventual model.
\end{abstract}

Key words: Articular Cartilage, Compressive Deformation, Finite Element Analysis, Biphasic Theory, Depth-Dependence, Collagen Fiber

\section{Introduction}

The human synovial joint possesses superior load-supporting and articulating functions with very low friction and low wear. Articular cartilage tissue plays an important role to maintain this function through whole life. Cartilage has high water content from 70 to $80 \%$ and shows biphasic behaviors in which both solid and fluid properties should be considered.

One of the representative diseases on the synovial joints particularly for elder persons is osteoarthritis. Osteoarthritis causes degeneration and destruction of articular cartilage, 
which lead to the disturbance of motility. Articular cartilage degenerated by osteoarthritis decreases a load-buffering capacity and leads to the bone deformation, the formation of osteophytes, and the subchondral bone induration and thickening. Especially in joints of lower extremities, under high load of several times as body weight in daily activities, mechanical factors are believed to play a crucial role in the pathogenesis of osteoarthritis.

As a new treatment method, tissue engineering has attracted attention in recent years. At the present stage, however, the mechanical function of tissue-engineered cartilage has not reached the level of native cartilage. It is noted that appropriate mechanical stimulation in culture process promotes extracellular matrix synthesis and leads to better function. Therefore, it is necessary to study the optimum conditions of mechanical stimulation.

Gaining better understanding of the mechanical and functional environment around chondrocytes is crucial to the clarification of the mechanism of osteoarthritis pathogenesis, the assessment of mechanical properties of the regenerated cartilage, and the clarification of the optimum condition of mechanical stimulation on the metabolism of chondrocytes.

The purpose of this study is to consider an influence of the collagen fiber network in addition to the depth-dependence in elastic modulus of cartilage. As an approach, first, we performed compression test of the cartilage tissue under unconfined condition in similar method to the previous study (Hosoda et al. ${ }^{(1)}$ ). Next, we created FE model for articular cartilage and applied to simulate actual behaviors based on biphasic theory (Mow et al. ${ }^{(2)}$ ).

\subsection{Articular cartilage tissue}

Articular cartilage is composed of chondrocytes and an extracellular matrix as shown in Fig. $1^{(1)}$. Extracellular matrix is mainly composed of proteoglycan and collagen, and produced by chondrocytes to provide scaffold to chondrocytes and support a load to cartilage. Proteoglycan is highly hydrophilic and therefore can store a large amount of water and contribute to the biphasic property. The hydrated proteoglycan is distended but confined by collagen network, and thus it supports the compressive load, while the collagen is given under pulling coercively.

Alexopoulos et al. ${ }^{(3)}$ investigated an influence of elastic modulus distribution in extracellular matrix on pericellular matrix. They calculated the elastic modulus about each position on depth direction from the unconfined compression test and showed that the strain in cartilage tissue is decreased with the depth. In our previous study ${ }^{(1)}$, the importance of depth-dependence of elastic modulus was exhibited in compression tests and biphasic finite element analysis. In this study, the effectiveness of collagen network (Fig.1) in addition to depth-dependence of elastic modulus is evaluated.

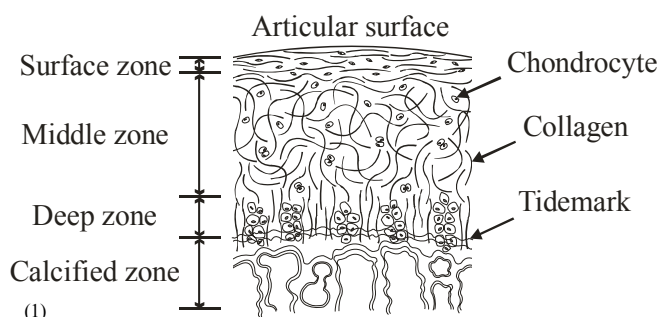

Fig.1 Cross-section diagram of cartilage tissue ${ }^{(1)}$

\subsection{Mechanical behavior of cartilage tissue under compression}

The time-dependent compressive behavior of cartilage becomes important in actual compression. If the load acts on the tissue rapidly, the tissue does not have enough time to exude the interstitial fluid and thus presents peak stress as shown in Fig. 2. When cartilage is kept under compression at the definite position, the stress relaxation behavior is observed, accompanied with gradual fluid flow from cartilage tissue. It is indicated in compression that the permeability of cartilage becomes lower with an increase in compression strain 
(Jurvelin et al. ${ }^{(4)}$ ). In our unconfined compression test of $10 \sim 15 \%$ strain at $1000 \mu \mathrm{m} / \mathrm{s}$ (at strain rate $0.33-1.01 / \mathrm{s}$ ), the peak stress ranged from $0.71 \mathrm{MPa}$ to $3.76 \mathrm{MPa}$. To understand the mechanotransduction in chondrocytes in cartilage, the actual time-dependent and depth-dependent stress and strain around chondrocytes should be clarified.

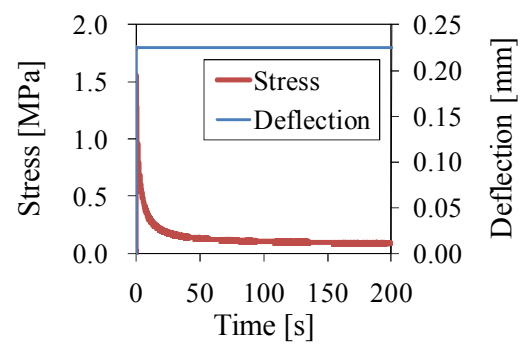

Fig. 2 Compressive behavior of articular cartilage of $1.5 \mathrm{~mm}$ thickness to definite compressive deflection (compression ratio: $15 \%$ )

\subsection{Composition of cartilage and biphasic theory}

Cartilage tissue is composed of chondrocytes and extracellular matrix with high water content and thus has biphasic properties. Biphasic theory is developed by Mow et $\mathrm{al}^{(2)}$. In this theory, cartilage tissue is modeled by acquiring the macroscopic tissue characteristics, and it is assumed that the soft tissue with high water content is composed of two types of phases, i.e., the solid phase and the fluid phase. And, the fluid phase is able to flow at some level through the solid phase composed of porous material. Because this fluid flux makes resistance to compressive load in cartilage, the whole tissue presents viscoelastic behavior. This biphasic theory is appropriate to express the stress relaxation behavior and the creep phenomenon. Thus, in this study, we conducted biphasic finite element analyses.

\section{Experimental methods for compression tests}

\subsection{Materials for compression tests}

Osteochondral explants were harvested from distal end of femur in porcine knee (6 to 7 months old). A $3 \mathrm{~mm}$ cylindrical core was punched from each osteochondral explant and then cut in half perpendicular to the articular surface with a scalpel blade (Fig. 3). The samples were washed by PBS (Phosphate Buffered Saline; pH7.4, Invitrogen Corporation). Then, living cells as markers were stained with Calcein-AM $1 \mu \mathrm{L}$ (Molecular Probes) in $1000 \mu \mathrm{L}$ PBS. The samples were incubated for $30 \mathrm{~min}$ at $37{ }^{\circ} \mathrm{C}$ and washed by PBS.

\subsection{Compression tester and experimental method}

The compression tester shown in Fig. 4 is located on the stage of the confocal laser scanning microscope (CLSM, BIO-RAD, Radiance2000). The tester is constructed by impermeable alumina ceramic compressing plates, load cell, gap sensor for measuring the displacement of compressing plate, and linear actuator. Compression test is performed on the cover glass. The compressive behavior can be observed by object lens of CLSM. In this loading system the load cell will restore according to actual decreasing load during stress relaxation, and therefore the position of load cell should be adjusted. The position accuracy of $0.2 \mu \mathrm{m}$ and the maximum speed of $4000 \mu \mathrm{m} / \mathrm{s}$ could be appropriately controlled by the feed back system for the displacement of compressing plate.

First, a semi-cylindrical sample was set up on the cover glass, and soaked with PBS to keep it from drying out. The initial position was the position that the sample has contact with the impermeable compression plate and detective load of about $0.1 \mathrm{~N}$. The thickness of the cartilage tissue was estimated from cartilage surface to tidemark (Fig.1).

Under unconfined condition, the cartilage specimen was compressed to the defined total deformation at a constant velocity. After that, the position was kept during stress relaxation phase for $20 \mathrm{~min}$. Peak stress was observed immediately after compression as shown in Fig. 2. During compression tests, the compressive load was monitored and fluorescence images were continuously acquired. 
In this study, we regulate that semi-cylindrical specimen is nearly uniaxially compressed at equilibrium after stress relaxation in unconfined compression test and discuss mainly about the case of compression velocity of $200 \mu \mathrm{m} / \mathrm{s}$ and the total compression ratio of $10 \%$.

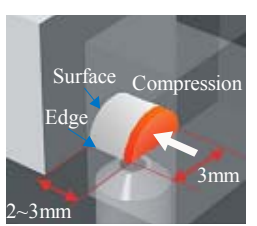

Fig.3 Test specimen in compressive device.

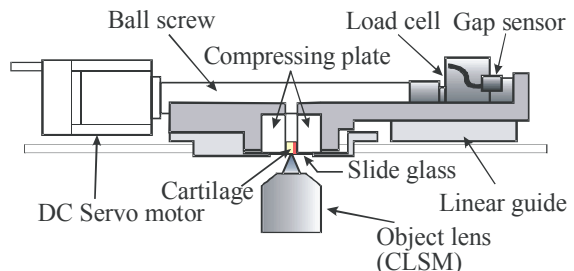

Fig.4 Compression tester located on microscope stage.

\subsection{Elastic modulus distribution depending on the depth}

Previously ${ }^{(1)}$, we performed unconfined compression test of articular cartilage at definite total deformation in compression tester located on the stage of CLSM. Then, the local compression strain is calculated from the change in distance between the corresponding living cells stained with Calcein-AM in the fluorescence images before compression and at equilibrium. We derived the elastic modulus distribution function using inverse relationship between strain and elastic modulus for nearly uniaxial compression.

The elastic modulus depending on the depth is described by Eq. (1), where $E_{0}$ and $z$ are average elastic modulus of solid phase at equilibrium and depth direction distance from the surface, respectively. In this study, we applied $0.26 \mathrm{MPa}$ as the average elastic modulus $E_{0}$ of solid phase at equilibrium. The elastic modulus is plotted against relative position $z / h$ in depth direction for the cartilage thickness of $h$ in Fig. 5. The surface side is depicted as 0 , and the deep zone end side is 1 .

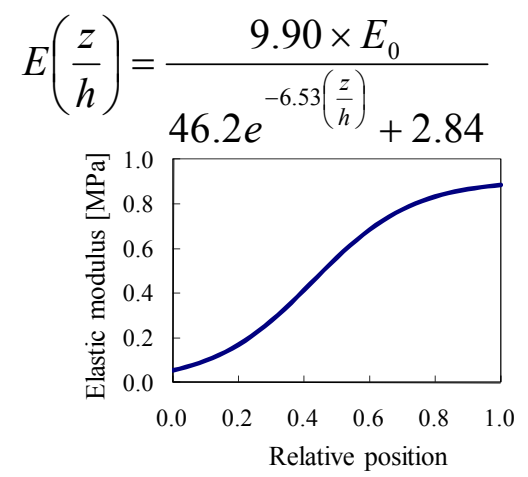

\section{Finite element analysis of articular cartilage model and boundary conditions}

\subsection{Finite element model}

General-purpose finite element analysis software (ABAQUS v.6.5) was used to analyze the biphasic model (Wu et al. ${ }^{(5)}$ ).

The shape of articular cartilage model is cylinder that has $1.5 \mathrm{~mm}$ radius and $1.4 \mathrm{~mm}$ height. We use axisymmetric model using axisymmetric solid element (CAX4P) that corresponds to 4-node, bilinear displacement and pore pressure, i.e., a biphasic element. The size of each element corresponds to $20 \mu \mathrm{m}$ x $20 \mu \mathrm{m}$.

In this model, the upper $10 \%$ of cartilage tissue is surface zone, the lower $20 \%$ of the tissue is deep zone and the middle zone is located between surface and deep zones. And the upper $10 \%$ of surface zone is defined as a superficial zone.

We noticed the function of collagen network. When proteoglycan supports compression loading, collagen fiber supports tension loading coercively because collagen network confines proteoglycan. So we created cartilage models. These models did not replace collagen fibrosis purely by spring element. The feature of composite body of proteoglycan and collagen network was modeled using biphasic element and spring elements. In this 
study, as shown in Fig. 6, we connect nodes of biphasic element in a horizontal direction by the spring element (SPRINGA) and specified that spring element affects only tensile deformation. In this study, a linear constant property was provided to a spring element. In the first model, we assumed that a spring element inhabits uniformly ( $\mathrm{Li}$ et al. ${ }^{(6)}$ ). In the second and third models, the depth-dependence of spring property is considered.

The void ratio $e$ is provided for ABAQUS by the following equation.

$$
e=\frac{d V_{v}}{d V_{g}+d V_{t}}
$$

Where $d V_{v}$ is the volume of voids, $d V_{g}$ is the volume of grains of solid material, and $d V_{t}$ is the volume of trapped wetting liquid.

In this study, we assumed that water content of $80 \%$ included in cartilage tissue is the volume of voids and other $20 \%$ is sum of the volume of grains of solid material and the volume of trapped wetting liquid. Thus, the void ratio is 4 .

Poisson's ratio and permeability of solid phase were assumed as constant and used literature data shown in Table.1 (Guilak et al. ${ }^{(7)}$ ).

In this study, average elastic modulus of solid phase at equilibrium is $0.26 \mathrm{MPa}$. The analysis was conducted for test duration of $1200 \mathrm{~s}$.

\begin{tabular}{|c|c|c|}
\hline $\begin{array}{c}\text { Elastic modulus } \\
\text { of solid phase, } E_{0}[\mathrm{MPa}]\end{array}$ & $\begin{array}{c}\text { Poisson's ratio } \\
\text { of solid phase, } v\end{array}$ & $\begin{array}{l}\text { Fig.6 Finite element and arrangement } \\
\text { of spring elements. } \\
\text { SPRINGA: Spring element affects only } \\
\text { tensile deformation. } \\
k\left[\mathrm{~m}^{4} / \mathrm{N} \cdot \mathrm{s}\right]\end{array}$ \\
\hline 0.26 & 0.125 & $2.0 \times 10^{-15}$ \\
\hline
\end{tabular}

\subsection{Boundary conditions}

To compare experimental result with finite element analysis (FEA), the boundary conditions for FEA were given to correspond to the compression test. FEA simulation of compression test was performed under compression velocity of $200 \mu \mathrm{m} / \mathrm{s}$ and compression ratio of $10 \%$. Thus, amount of compression, compression velocity and compression time to definite deflection were $0.14 \mathrm{~mm}$ as $10 \%$ of thickness, $200 \mu \mathrm{m} / \mathrm{s}$ and $0.696 \mathrm{~s}$, respectively.

We assumed that there is rigid body plate on the cartilage tissue model and gave compressive displacement. The friction coefficient between rigid plate and top surface was assumed as 0 . All nodes of top surface were compressed at displacement of $0.14 \mathrm{~mm}$ by the plate and the transformation in $r$ direction (parallel to surface) was unconfined. All nodes of lower impermeable boundary were confined and the displacements of the nodes in both $r$ and $z$ directions were specified as 0 . To simulate impermeable compressing plates in compression tester, water was assumed to seep only through side of cylinder.

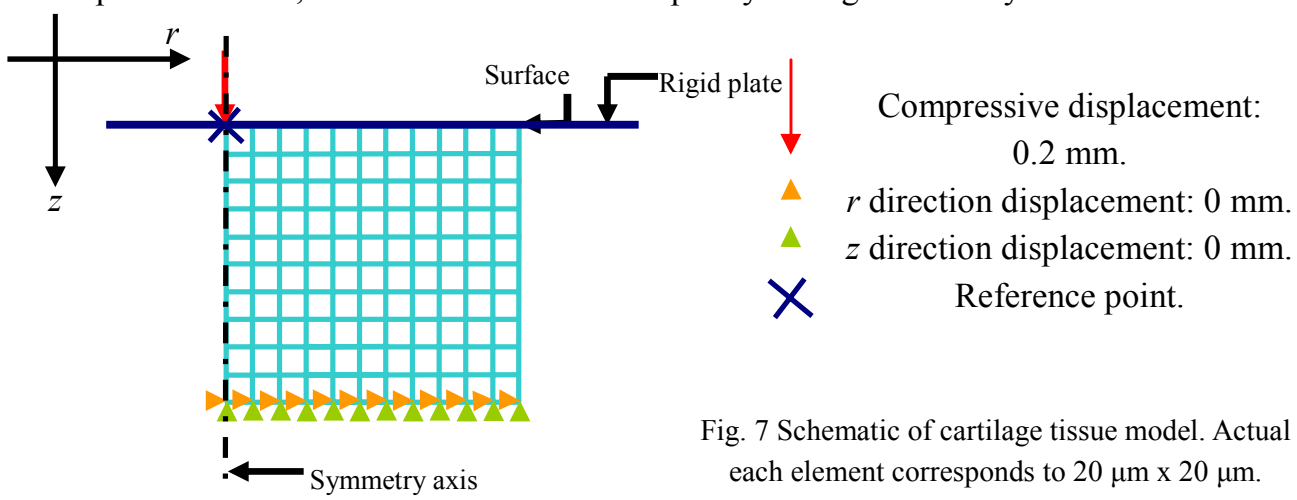




\section{Results of finite element analyses}

4.1 Depth-dependent elastic modulus and uniform linear spring element model (Model 1)

Depth-dependent elastic modulus (Fig. 5) was determined based on the local strain of solid phase at equilibrium ${ }^{(1)}$. Instantaneous fibrillar elastic modulus $K_{\text {total }}(t)$ of spring in Model 1 was changed so that the total load capacity of FEA value corresponded to experimental one (Fig. 8). Total load capacity and deformed profiles immediately after compression of specimen and FEA simulation on Model 1 are shown in Fig. 9 and Fig. 10, respectively. Total load capacity as average stress of FE model can be obtained by dividing reaction force detected for rigid body on cartilage tissue model by surface area. By controlling the time-dependent spring constant (Fig.8) which changes as an eventual phenomenon caused by outward flow of fluid, the total load capacity of FEA simulation based on biphasic theory could be conformed to the experimental value. However, the deformed profile immediately after compression of the Model 1 showed semi-barreled profile and disaccorded with experimental one. Therefore, the depth-dependence of spring property is considered in the next model.

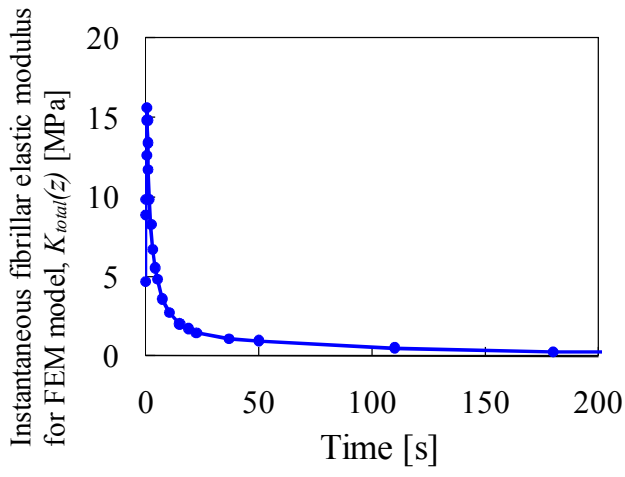

Fig. 8 Time-dependent behavior of linear spring element (Model 1).

Fig. 10 Cross-section diagram of cartilage specimen and FE model immediately after compression. Blue and green dashed line indicates columnar side edge of cartilage specimen and FE model, respectively. (a) Cartilage specimen. (b) Model 1.

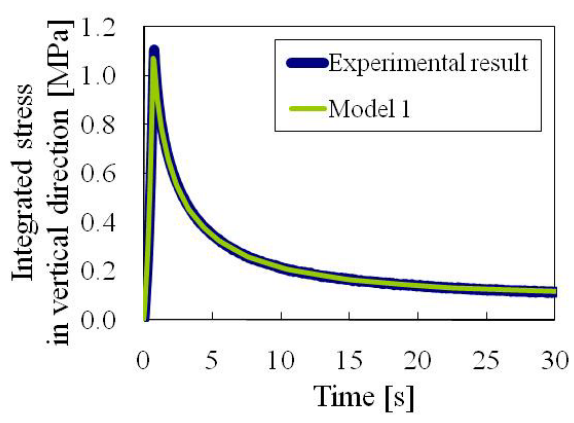

Fig. 9 Total load capacity from beginning of compression to $200 \mathrm{~s}$ (Model 1).

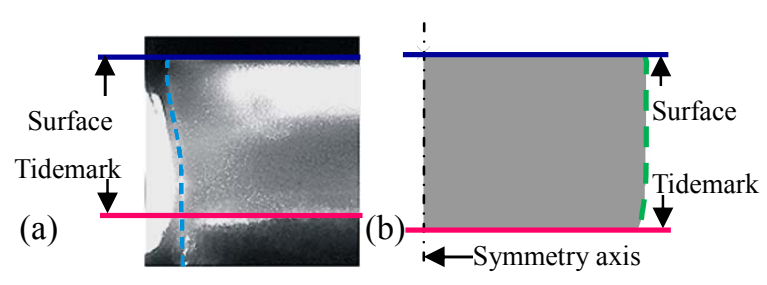

4.2 Cartilage tissue models considering spring element having depth-dependence

4.2.1 Cartilage tissue model considering distribution of the content fraction for horizontally oriented collagen fibrils based on tensile test result (Model 2)

As shown in Fig.1, the alignment of collagen fiber is different from surface to deep zone. Therefore, it is thought that function caused by collagen fiber also has depth-dependence. Li et al. ${ }^{(8)}$ defined Eq. (3) based on tensile test result of cartilage tissue.

$$
\omega=1+0.6 \frac{z}{h}-1.6\left(\frac{z}{h}\right)^{2}
$$

$\omega$ notes distribution of the content fraction from surface to deep zone for horizontally oriented collagen fibrils, where $z$ and $h$ are distance from the top surface and height of FE model, respectively. We plotted Eq. (3) in Fig. 11.

If the compression load is provided to cartilage tissue, the hydrated proteoglycan is distended but confined by collagen network, and thus it supports the compressive load, while the collagen is given under pulling coercively. Therefore, when cartilage cylinder is 
given under axial compressive force, it is expected that the content fraction for horizontally oriented collagen fibrils controls radial deformation. Thus, we created cartilage tissue model considering distribution of the content fraction for horizontally oriented collagen fibrils based on tensile test result (Model 2). In the same way as Model 1, instantaneous fibrillar elastic modulus $K_{\text {total }}(t)$ of Model 2 was changed so that total load capacity of FEA value corresponded to experimental one (Fig. 12). Depth-dependence of spring constant of each spring was defined considering Eq. (3).

As shown in Fig. 13, the total load capacity in FEA simulation was conformed to the experimental value by controlling spring constant. However, deformed profiles immediately after compression of specimen and FEA simulation on Model 2 indicated exact opposite tendencies as shown in Fig. 14. In the case of Model 2, the radial strain in surface zone is smaller than in deep zone. On the other hand, actual radial strain of specimen in surface zone is larger than deep zone. Thus, it is clear that the depth-dependence of collagen network affects deformed profiles. Therefore, in the third model, the appropriate depth-dependence of spring property is reconsidered.

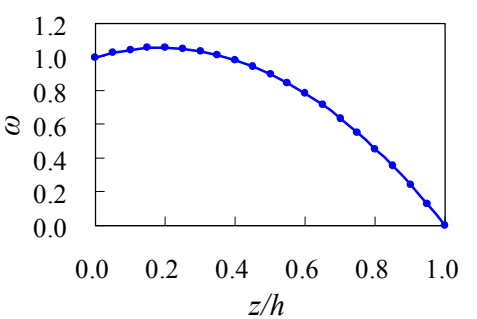

Fig.11 Depth-dependence of collagen network proposed by $\mathrm{Li}$ et al ${ }^{(8)} \omega$ notes distribution of the content fraction from surface to deep zone for horizontally oriented collagen fibrils. $z$ and $h$ are distance from the top surface and height of FE model.

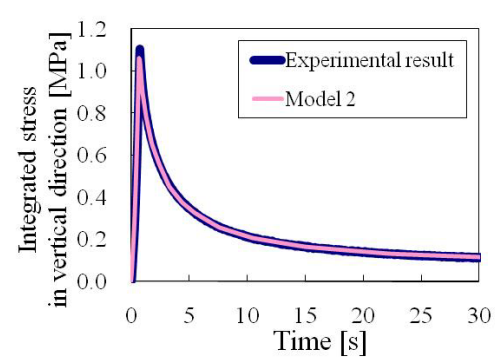

Fig. 13 Total load capacity from beginning of compression to $200 \mathrm{~s}$ (Model 2).

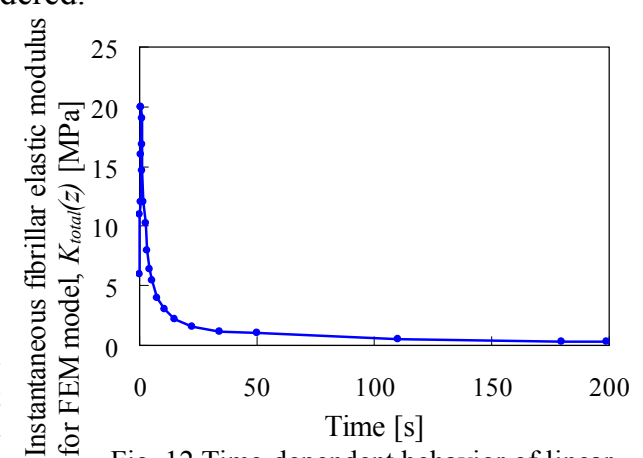

Fig. 12 Time-dependent behavior of linear spring element (Model 2).
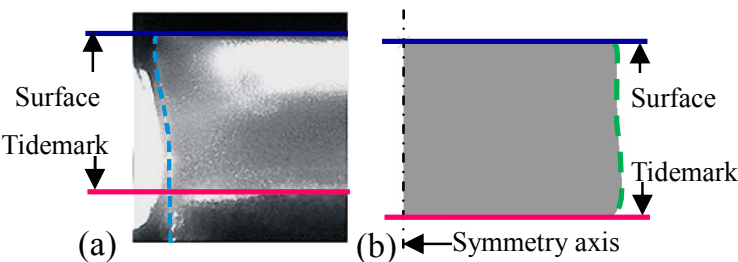

Fig. 14 Cross-section diagram of cartilage specimen and FE model immediately after compression. Blue and green dashed line indicates columnar side edge of cartilage specimen and FE model, respectively. (a) Cartilage specimen. (b) Model 2.

\subsubsection{Cartilage tissue model considering depth-dependent function caused by} combined property of collagen network and proteoglycan (Model 3)

In general, in the case of tensile test using cartilage tissue specimens with definite thickness cut perpendicular to depth direction, the tensile stiffness of surface is higher than that of deep zone (Kempson et al. ${ }^{(9)}$ ). From this result, when cartilage tissue specimen was given under tensile load, it is thought that depth-dependence of collagen network gives greater resistance in surface zone than deep zone. Buckley et al. ${ }^{(10)}$ performed shear test using cartilage tissue specimen and searched distribution of shear strain and shear stiffness of depth direction. The result indicated that maximum shear strain occurs in surface, while shear strain has few change from middle zone to deep zone. Additionally, Krishnan et al. ${ }^{(11)}$ sliced disk-shaped cartilage specimen into 4 layers to depth direction, and performed compression tests under confined and unconfined constant total deformation conditions, and studied mechanical properties of each layer. Thus, they calculated aggregate modulus in both compression and tension. In their tests, the tensile modulus showed higher value in surface layer and the compressive modulus showed higher one in deep layer. 
In this study creating FE model based on compression test result, it is necessary to reconsider effective characteristics of collagen network when cartilage tissue is compressed. As already discussed, in deformed profiles after compression of a semi-cylindrical cartilage specimen, the radial strain of surface zone is larger than in deep zone. This result suggests that not simple tensile property of collagen fibers but the combined property of collagen network and proteoglycan in extracellular matrix can restrict the radial deformation in compression of cartilage. The radial strain appears to be controlled by depth-dependent spring effect as modeled in Fig. 15, as described in detail below.

In cartilage tissue immediately after compression at high strain rate, it has not enough time for intrinsic fluid to flow out of cartilage, and high fluid pressure supports considerable part of total load. During total deflection is maintained after compression, the intrinsic fluid flow gradually occurs. Intrinsic fluid moves to the side of cylinder along gradient of pore pressure, because water was assumed to seep only through the side of cylinder in this model. In stress relaxation process, the intrinsic fluid flow gradually occurs and it is expected that the resistance to radial strain afforded by combined structure of collagen network and proteoglycan becomes weaker as shown in Fig. 16. During compression test, in surface where the elastic modulus of solid phase is lower than average value, the compressive strain of surface increases and the fluid flows through outer cylindrical boundary as time advances. On the other hand, in deep zone where the elastic modulus is higher, the compressive strain is lower than surface zone and the radial strain appears to maintain as small probably due to combined effect of collagen network and proteoglycan.

Thus, the depth-dependence of spring element modeling as Model 3 considering effectiveness of collagen network was defined as shown in Fig. 15 to conform the deformed profiles immediately after compression of FEA simulation to the experimental value. In the same way as Model 2, instantaneous fibrillar elastic modulus $K_{\text {total }}(t)$ of Model 3 was changed as total load capacity of FEM value corresponded to experimental one (Fig. 16). Depth-dependence of spring constant of each spring was defined considering Eq. (4).

$$
\psi=\frac{1}{0.22\left\{15\left[0.1 \times \exp \left(10\left(\frac{z}{h}\right)\right)+1\right]^{-1}+3\right\}}
$$

Total load capacity and deformed profiles immediately after compression of specimen and FEA simulation on Model 3 are shown in Fig. 17 and Fig. 18, respectively. In Model 3, both the total load capacity and deformed profile show satisfied solutions.

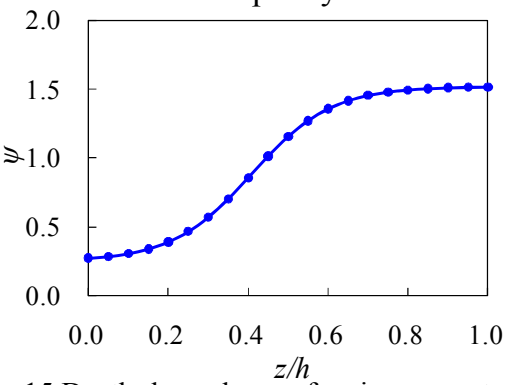

Fig. 15 Depth-dependence of spring property $\psi$ cluculated by Eq. (4). $z$ and $h$ are distance from the top surface and height of FE model.

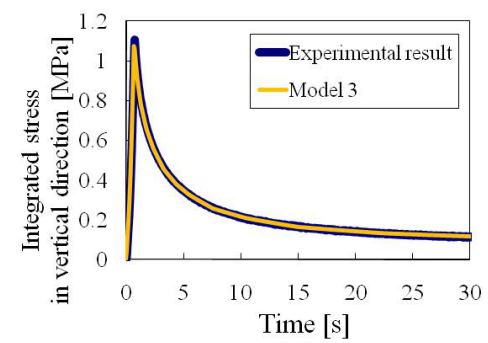

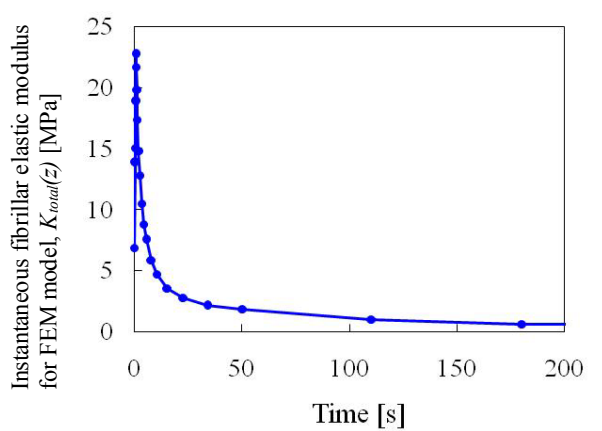

Fig. 16 Time-dependent behavior of linear spring element (Model 3).

Fig. 17 Total load capacity from beginning of compression to $200 \mathrm{~s}$ (Model 3). 


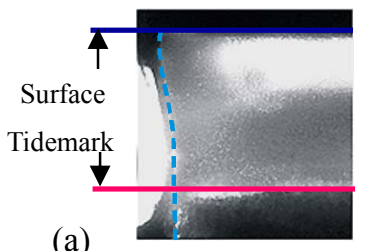

(a)

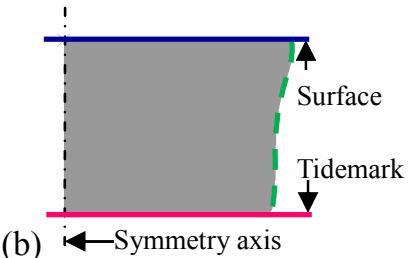

(b)
Fig. 18 Cross-section diagram of cartilage specimen and FE model immediately after compression. Blue and green dashed line indicates columnar side edge of cartilage specimen and FE model, respectively.

(a) Cartilage specimen. (b) Model 3.

\section{Comparison of internal behavior for Model 3}

\subsection{Temporal change in local compressive strain of axial direction in unconfined compression test}

Temporal change in local compressive strain of Model 3 is shown in Fig. 19. Representative 1 element was selected from each layer and its local strain was calculated based on axial displacement of node of element. In superficial zone, the local strain increases as time advances from approximately $20 \%$ immediately after compression to around $40 \%$ at equilibrium. In surface zone, it increases from approximately $20 \%$ to around $30 \%$ at equilibrium. In contrast, in middle zone, the local strain decreases from about $8 \%$ towards $6 \%$ at equilibrium as recovery. In deep zone, it recovers from about $4.4 \%$ as initial strain towards $3 \%$ at equilibrium.

Comparing FEM results to experimental ones ${ }^{(12)}$, these show common tendency that the axial compressive strain increases in superficial and surface zones, while axial compressive strain decreases in middle and deep zones with stress relaxation. Thus, it was clear that not only total load capacity and deformed profiles immediately after compression but also local strain of axial direction in FEA results for Model 3 corresponded to experimental ones.

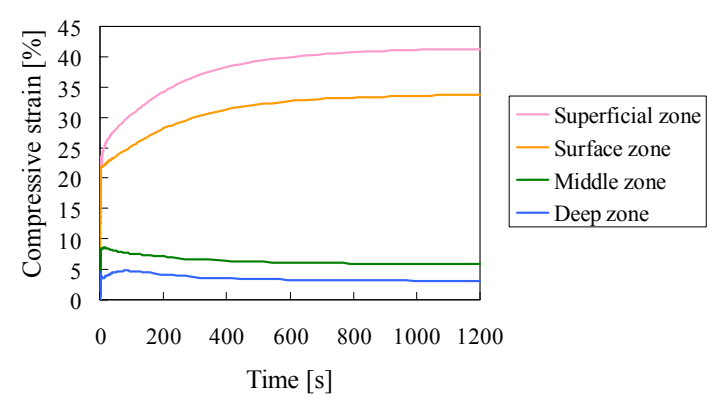

Fig. 19 Local axial compressive strain behavior of Model 3. Relative distance from surface are 0.014 (Superficial), 0.055 (Surface), 0.45 (Middle zone), and 0.905 (Deep zone).

\subsection{Intrinsic fluid behavior of finite element model.}

As previously explained, it was able to confirm that, in addition to the total load capacity and the deformed profiles immediately after compression, the local axial compressive strain calculated in Model 3 considering actual compressive behavior and configuration of cartilage tissue corresponded to experimental result. Therefore, it can be expected in Model 3 to conform intrinsic fluid flow of FE model to experimental one. Thus, the pore pressure and intrinsic fluid flow were examined using Model 3.

The deformed profile immediately after compression, the pore pressure, and the effective velocity of pore fluid are shown in Fig. 20 - Fig. 22. When a cylindrical cartilage specimen is compressed, the pressure gradient develops in radial direction from the central region of FE model to the side of cylinder. It is confirmed that the fluid flow occurs along this gradient. Immediately after compression, the intrinsic fluid flow is large according to high pressures gradient, and the compressive stress relaxes rapidly (Fig. 17). Changes in stress relaxation become slow gradually because the pore pressure gradient becomes low beyond $30 \mathrm{~s}$ from the beginning of compression

The effective velocity of pore fluid is plotted as direction of arrow line and length. Flux is the product of velocity and section area for fluid. Immediately after compression, the 
fluid flow occurs at all outer cylindrical area particularly in deep zone as shown in Fig. 20. As time advances, the fluid flow occurs in wider region of the model as indicated in Fig. 21. After a period of time (in Fig. 22), the fluid flow of surface tends to increase, while the fluid flow of deep zone tends to decrease. The changes in fluid flow are observed in upper half area as a result of change in pore pressure distribution. The fluid flows from the surface zone to the middle and deep zones are noticed at (3) and (4) in Fig. 22, which contributes to the recovery of compressive strain in middle and deep zones.

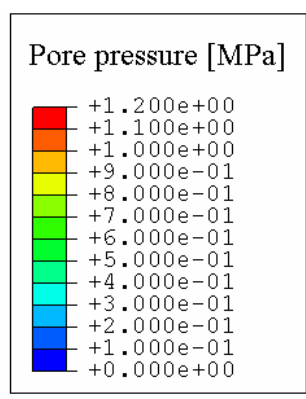

(a)

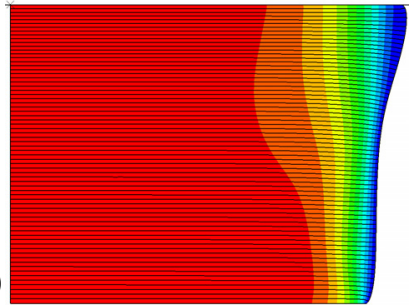

(b)
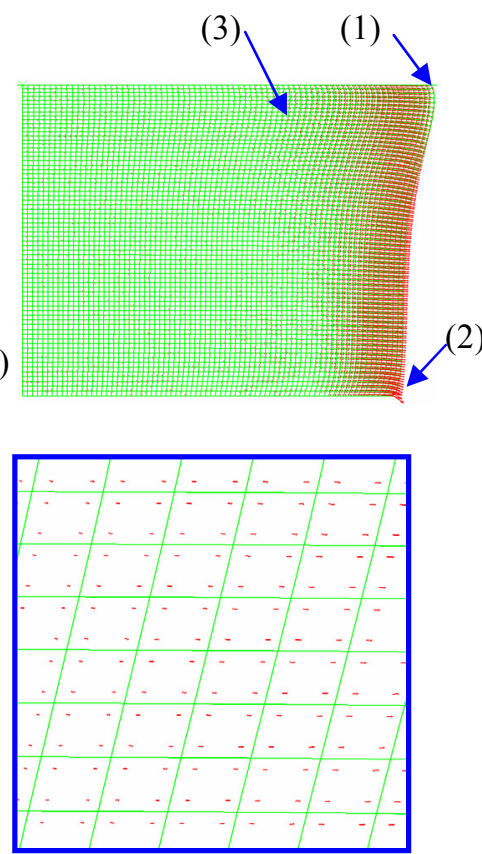

(3)

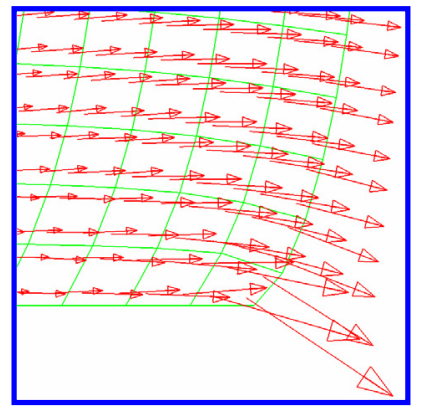

(2)
(1)

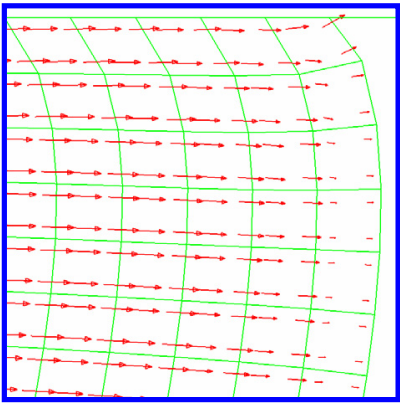

F pore pressure. (b) Effective velocity of pore fluid.

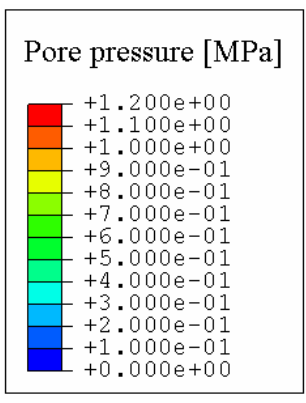

(a)

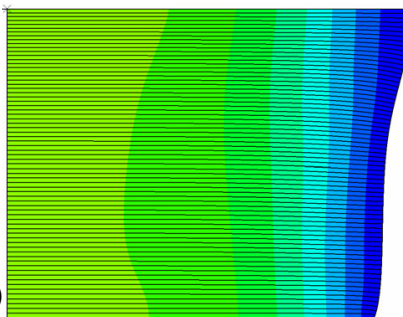

(b)

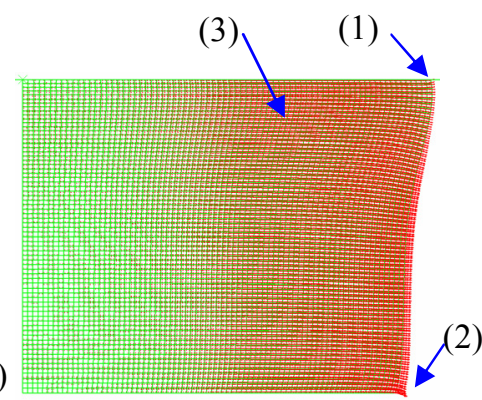

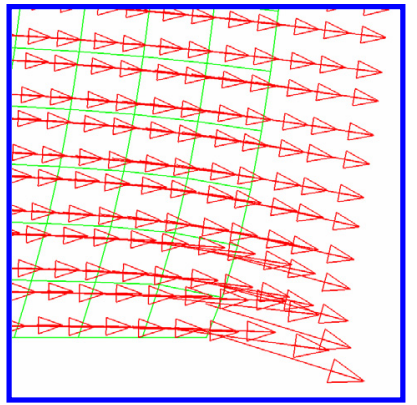

(2)

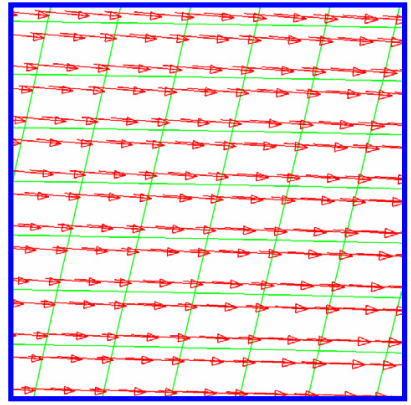

(3)

(1)

Fig. 21 Intrinsic fluid behavior of Model 3 at 2.009 s after compression. (a) Deformed shape and pore pressure. (b) Effective velocity of pore fluid. 
(a)
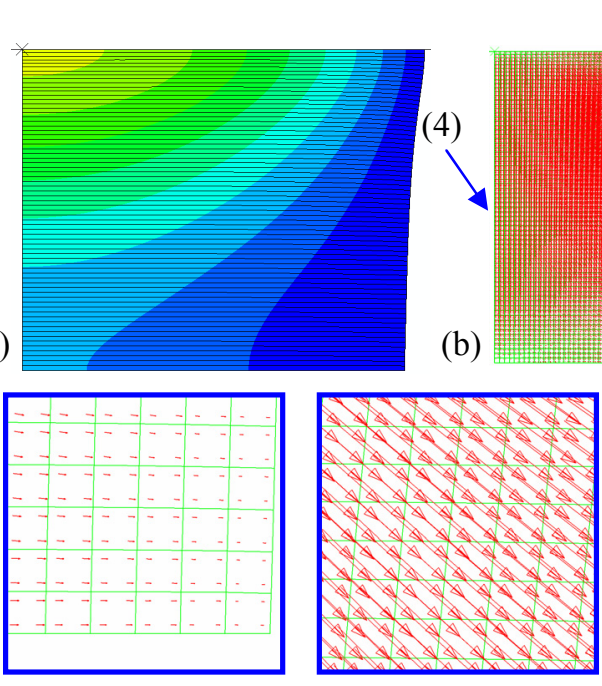

(1)

(3)
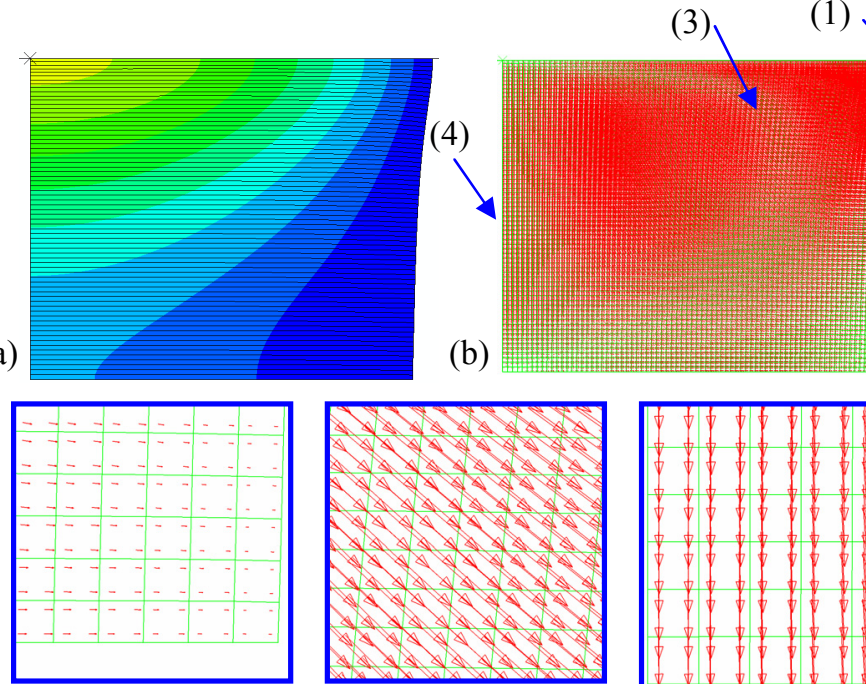

(2)
(3)

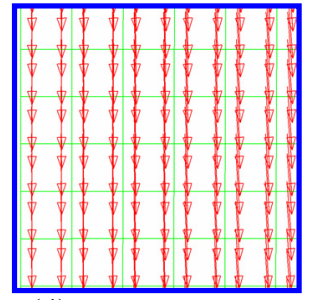

(4)

Fig. 22 Intrinsic fluid behavior of Model 3 at 212.4 s after compression. (a) Deformed shape and pore pressure. (b) Effective velocity of pore fluid.

\section{Discussion and conclusions}

In this study, we created cylindrical models of articular cartilage tissue using axisymmetric biphasic element and spring element, and performed the finite element analyses based on biphasic theory. Then, FEA results were compared with experimental ones in unconfined compression tests under constant total deformation. We created 3 types of models. Every FE model has depth-dependence of elastic modulus based on our previous formula, while the Poisson's ratio and permeability of solid phase were assumed as constant in literature data. Boundary conditions are also the same in all models.

By controlling spring constant, the total load capacity of Model 1 with the same spring element from surface to bottom corresponded to experimental value from beginning of compression to the end. However, the deformed profile of the Model 1 immediately after compression showed semi-barreled profile and disaccorded with experimental one.

Next, the depth-dependence of spring element was considered about Models 2 and 3. By controlling spring constant in these FE models, the total load capacity of both FE models corresponded to experimental one from beginning of compression to the end. However, the differences in depth-dependence of collagen network affected their deformed profiles immediately after compression.

In Model 2 like a model reported by Li et. al. ${ }^{(8)}$, in the case using a viewpoint that collagen fibers affect radial stiffness in surface zone than in deep zone as a result of considering distribution of the content fraction for horizontally oriented collagen fibrils based on tensile test result, the deformed profiles immediately after compression indicated an exact opposite tendency to experimental result. Li et. al. validated the total load capacity but did not examine the accordance in deformed profiles.

Therefore, we created a new model reflecting the deformation behavior during compression (Model 3). In considering non-uniformity of configuration of cartilage tissue from surface to deep zone, we found that the combined effect of network of collagen fibers and proteoglycan enhances the radial stiffness in deeper zone than in surface zone in compression test (Fig. 15). As a result, the deformed profile immediately after compression of Model 3 corresponded to the actual profile (Fig. 18).

As a result of comparison of local strain obtained by experimental result to FEA result of Model 3, it was clarified that not only the total load capacity and deformed profiles immediately after compression but also the time-dependent local strain of axial compressive direction corresponded to experimental result (Fig. 19). 
Then, the pore pressure and intrinsic fluid flow were examined using FE model 3. Immediately after compression, the intrinsic fluid significantly seeps through the side of cylinder according to high pressure gradient, and thus stress relaxes rapidly. As time advances, the fluid flow of surface zone increases, while the fluid flow from deep zone is reduced. The compressive strain of surface increases and the radial deformation decreases because the fluid flow from surface increases. In contrast, the compressive deformation in middle and deep zones is recovered. Thus, it is noticed that this intrinsic fluid flow from immediately after compression to equilibrium affects temporal changes in local strain.

As future studies, the in situ measurement of fluid flow in cartilage tissue and the consideration of nonlinearity in permeability and spring property are expected.

\section{Acknowledgement}

Financial support was given by the Grant-in-Aid for Scientific Research (A) of Japan Society for the Promotion of Science (21246029).

\section{References}

(1) N Hosoda, N Sakai, Y Sawae, T Murakami, Depth-dependence and time-dependence in mechanical behaviors of articular cartilage in unconfined compression test under constant total deformation, Journal of Biomechanical Science and Engineering, Vol.3, No.2, pp.209-220, 2008

(2) V C Mow, S C Kuei, W M Lai, C G Armstrong, Biphasic creep and stress relaxation of articular cartilage in compression theory and experiment, Journal of Biomechanical Engineering, Vol.102, pp.73-84, 1980

(3) L G Alexopoulos, L A Setton, F Guilak, The biomechanical role of the chondrocyte pericellular matrix in articular cartilage, Acta Biomaterialia, Vol.1, pp.317-325, 2005

(4) J S Jurvelin, M D Buschmann, E B Hunziker, Mechanical anisotropy of the human knee articular cartilage in compression, Proceedings of the Institution of Mechanical Engineers, Part H: Journal of Engineering in Medicine, Vol.217, pp.215-219, 2003

(5) $\mathrm{J} \mathrm{Z} \mathrm{Wu}, \mathrm{W}$ Herzog, M Epstein, Evaluation of the finite element software ABAQUS for biomechanical modeling of biphasic tissues, Journal of Biomechanics, Vol.31, pp.165-169, 1998

(6) L P Li, J Soulhat, M D Buschmann, A Shirazi-Adl, Nonlinear analysis of cartilage in unconfined ramp compression using a fibril reinforced poroelastic model, Clinical Biomechanics, Vol.14, pp.673-682, 1999

(7) F Guilak, V C Mow, The mechanical environment of the chondrocyte: a biphasic finite element model of cell-matrix interactions in articular cartilage, Journal of Biomechanics, Vol.33, pp.1663-1673, 2000

(8) L P Li, M D Buschmann, A Shirazi-Adl, A fibril reinforced nonhomogeneous poroelastic model for articular cartilage: inhomogeneous response in unconfined compression, Journal of Biomechanics, Vol.33, pp.1533-1541, 2000

(9) G E Kempson, H Muir, C Pollard, M Tuke, The tensile properties of the cartilage of human femoral condyles related to the content of collagen and glycosaminoglycans, Biochimica et Biophysica Acta, Vol.297, pp.456-472, 1973

(10) M R Buckley, J P Gleghorn, L J Bonassar, I Cohen, Mapping the depth dependence of shear properties in articular cartilage, Journal of Biomechanics, Vol.41, pp.2430-2437, 2008

(11) R Krishnan, S Park, F Eckstein, G A Ateshian, Inhomogeneous cartilage properties enhance superficial interstitial fluid support and frictional properties, but do not provide a homogeneous state of stress, Journal of Biomechanical Engineering, Vol.125, pp.569-577, 2003

(12) T Murakami, N Sakai, Y Sawae, K Tanaka and M Ihara, Influence of Proteoglycan on Time-Dependent Mechanical Behaviors of Articular Cartilage under Constant Total Compressive Deformation, JSME International Journal, Series C, Vol.47, pp1049-1055, 2004 